\title{
Increased Postprandial Colonic Motility and Autonomic Nervous System Activity in Patients With Irritable Bowel Syndrome: A Prospective Study
}

\author{
Yukari Tanaka, ${ }^{1,2 *}$ Motoyori Kanazawa, ${ }^{2,3}$ Olafur S Palsson, ${ }^{3}$ Miranda A Van Tilburg, ${ }^{3}$ Lisa M Gangarosa, ${ }^{3}$ Shin Fukudo, ${ }^{2}$ Douglas A \\ Drossman, ${ }^{3}$ and William E Whitehead ${ }^{3}$ \\ ${ }^{I}$ Tohoku Medical Megabank Organization, Tohoku University, Sendai, Japan; ${ }^{2}$ Department of Behavioral Medicine, Tohoku University Graduate \\ School of Medicine, Sendai, Japan; and ${ }^{3}$ Center for Functional GI and Motility Disorders, University of North Carolina at Chapel Hill, NC, USA
}

\section{Background/Aims}

The prevalence and severity of irritable bowel syndrome (IBS) declines with age, but the cause of this is unknown. This study tested 2 hypotheses: (1) autonomic nervous system responses to eating and bowel distention, measured by heart rate variability (HRV), differs by age in IBS patients and (2) HRV is correlated with colonic motility and IBS symptoms.

\section{Methods}

One hundred and fifty-six Rome III positive IBS patients and 31 healthy controls underwent colonic manometry with bag distention in the descending colon, followed by ingestion of an 810-kcal meal. HRV, evaluated by low frequency (\%LF; 0.04-0.15 Hz) component, high frequency (\%HF; $0.15-0.40 \mathrm{~Hz}$ ) component, and the LF/HF ratio, was measured during colonic distention and after the meal. Motility index and subjective symptom scores were simultaneously quantified.

\section{Results}

Both colonic distention and eating decreased \%HF and increased the LF/HF ratio, and both indices of autonomic nervous system correlated with age. In IBS patients, \%HF negatively correlated with the postprandial motility index after adjusting for age. The \%HF and LF/HF ratios also correlated with psychological symptoms but not bowel symptoms in IBS patients.

\section{Conclusion}

Decreased vagal activity is associated with increase in age and greater postprandial colonic motility in patients with IBS, which may contribute to postprandial symptoms.

(J Neurogastroenterol Motil 2018;24:87-95)

Key Words

Autonomic nervous system; Gastrointestinal Motility; Irritable bowel syndrome; Postprandial period

Received: December 22, 2016 Revised: May 15, 2017 Accepted: August 16, 2017

(.) This is an Open Access article distributed under the terms of the Creative Commons Attribution Non-Commercial License (http://creativecommons. org/licenses/by-nc/4.0) which permits unrestricted non-commercial use, distribution, and reproduction in any medium, provided the original work is properly cited.

*Correspondence: Yukari Tanaka, MD, PhD

Tohoku Medical Megabank Organization, 2-1 Seiryo-machi, Aoba-ku, Sendai 980-8573, Japan Tel: +81-22-717-8162, Fax: +81-22-717-8214, E-mail: y-tanaka@med.tohoku.ac.jp 


\section{Introduction}

Irritable bowel syndrome (IBS) is a chronic gastrointestinal disorder which is defined by recurrent abdominal pain and/or abdominal discomfort associated with changes in the consistency or frequency of bowel movements. ${ }^{1}$ Around $5-15 \%$ of adults suffer from $\mathrm{IBS}^{2,3}$ and it is more prevalent in adolescence or in early adulthood than in older adults. ${ }^{4}$ The pathophysiology of IBS is presumed to be a disorder of the brain-gut link. ${ }^{1}$ Psychological stress exacerbates the cardinal symptoms of IBS. ${ }^{4,5}$ This phenomenon is believed to be mediated by activation of emotional brain networks and changes in activities of the autonomic nervous system (ANS) and neuroendocrine system, which may trigger abnormal colonic motility and visceral hypersensitivity. ${ }^{6}$ These facts suggest that age-dependent ANS function may be one of the key factors of IBS pathophysiology.

Heart rate variability (HRV) is often used as a biomarker for ANS activity. ${ }^{7}$ The parasympathetic motor pathways, which consist of the vagus nerves, modulate the gastrointestinal reflexes, ${ }^{8}$ and IBS patients are reported to have greater parasympathetic withdrawal compared to the baseline after meal ingestion. ${ }^{9,10}$ IBS patients often exhibit greater postprandial abdominal pain, discomfort, urge, and defecation, and greater colonic motility (gastro-colonic reflex) compared to healthy controls. ${ }^{11-13}$ Other studies have shown that both the sympathetic and parasympathetic nervous system responses to stimuli are attenuated in older subjects. ${ }^{14,15}$ Findings include a decline in baroreceptor sensitivity ${ }^{16}$ and altered adrenergic receptor function. ${ }^{17}$ Epidemiological studies have also reported age-related decreases in the prevalence of IBS, ${ }^{18}$ but there has been no study of age-dependent ANS changes in IBS. Furthermore, there have been no reports on the relationship among age-dependent ANS function, postprandial colonic motility, visceral pain thresholds, and symptoms in IBS.

The purpose of this study was to investigate the associations between HRV and sensorimotor function of the colon, and how these correlations are affected by age. We tested the following hypotheses: (1) the sympathetic and vagal components in response to meals are different by age in patients with IBS, and (2) HRV during the postprandial period correlated with colonic motility, visceral pain thresholds, and IBS symptoms.

\section{Materials and Methods}

\section{Subjects}

This was a prospective study. Subjects and study design have been previously reported, ${ }^{19,20}$ but the hypothesis that there are age-related differences in ANS activity and its response to meals and intraluminal distention has not been described previously. ${ }^{19,20}$ Subjects were recruited via advertisements or physician referrals and screened by telephone. The study population consisted of 156 patients who fulfilled the Rome III criteria for IBS, had received a physician diagnosis of IBS, and had current symptoms (abdominal pain or discomfort at least 3 days per month in the last 3 months). These subjects had no history of gastrointestinal surgery other than an appendectomy or cholecystectomy, no inflammatory bowel disease, celiac disease, lactose malabsorption, cardiovascular disease, or diabetes mellitus, and they were not pregnant at the time of study. Medication for IBS was stopped for 3 days before the experiment. However, antidepressants were discontinued 1 week before the procedure (with the consent of the patient's physician). Subtypes of IBS (diarrhea-predominant IBS [IBS-D], constipation-predominant IBS [IBS-C], mixed IBS [IBS-M], and unsubtyped IBS [IBSU]) were classified by the predominant stool pattern according to the Rome III diagnostic classification.

The control population was recruited via advertisements and consisted of 31 subjects without any significant or recurring gastrointestinal symptoms. Exclusion criteria for healthy controls were average stool frequency of less than 3 per week or more than 3 per day, use of a laxative or antidiarrheal agent on more than 2 occasions over the prior year, history of alcohol or substance abuse, a psychiatric diagnosis, or any of the medical conditions listed above for the IBS patients. Sample size was calculated from the standard statistical design and previous study. ${ }^{21}$ The study was approved by the Institutional Review Board of the University of North Carolina (UNC) (IRBIS 01-1397), and all subjects provided written informed consent.

\section{Study Design}

Subjects were studied at the Clinical Research Center at the UNC for a 24- to 30-hour period. They were instructed to fast for at least 4 hours prior to reporting for admission. On the day of admission, a medical history, physical examination, and breath test for lactose intolerance and small intestinal bacterial overgrowth were completed. The IBS-Quality of Life (IBS-QOL), ${ }^{22}$ IBS Symptom 
Severity Scale (IBS-SSS), ${ }^{23}$ and Brief Symptom Inventory (BSI) which assesses psychological distress ${ }^{24}$ were administered on a computer terminal during the breath test. A low-fiber meal was consumed at approximately 5 PM. The day before experiment ended with a bowel cleanout consisting of $1.5 \mathrm{oz}$ of Fleet's phosphosoda enema administered at $6 \mathrm{PM}$ and repeated at $9 \mathrm{PM}$.

\section{Assessment of Heart Rate Variability}

Heart rate was recorded using continuous electrocardiography (ECG) with 3 electrodes attached to the chest. The 24-hour ECG data were processed through a ProComp + Analyzer (Thought Technology Ltd, Montreal, Quebec, Canada). HRV measurements were taken throughout the procedure, from baseline to postprandial period, using CardioPro 2.0 software (Thought Technology). The ECG was sampled at a frequency of $256 \mathrm{~Hz}$, each QRS complex was detected automatically by software, and R-R intervals were identified to quantify HRV. The Cardiopro software divided the recorded data into 5-minute periods. Data acquisition began after 10 seconds of barostat bag inflation. We analyzed the heart rate and inter-beat interval values for each period, and artifacts caused by body movements, extra beats, missed beats or premature atrial/ ventricular contractions were manually corrected or removed by using CardioPro software.

The ECG was recorded as a series of time intervals between beats (the R-R intervals) in 5-minute blocks. These beat-to-beat intervals were separated into 2 frequency ranges: a low frequency (LF) band of less than $0.15 \mathrm{~Hz}$ and a high frequency (HF) band of greater than $0.15 \mathrm{~Hz}$. Previously published data shows that the LF band $(0.04$ to $0.15 \mathrm{~Hz})$ reflects sympathetic activity and the $\mathrm{HF}$ band $(0.15$ to $0.40 \mathrm{~Hz})$ represents parasympathetic activity. The ratio of low frequency to high frequency reflects the sympatheticparasympathetic balance. $^{7,25}$

\section{Colonic Sensory and Motility Testing}

The study protocol was previously described in detail. ${ }^{26}$ All physiological and sensory testing was performed on day 2. On that morning, the barostat catheter with 4 small catheters used to measure pressures $2.5 \mathrm{~cm}$ and $5 \mathrm{~cm}$ from the proximal and distal edges of the bag (Model CT-BP600R; Mui Scientific, Mississauga, Ontario, Canada) was placed in the descending colon by advancing it over a guide wire placed by sigmoidoscopy. After placement, the position of the barostat catheter was confirmed by fluoroscopy. Following catheter placement, the subject rested for 90 minutes before examination. No sedation was used throughout the duration of the procedure. Subjects fasted until the test meal. During this time, the subjects were taught how to use the rating scales to describe the intensity of the sensations of pain and urgency to defecate using a 6-point scale. Sensory rating occurred at the end of each distention. Colonic sensory testing was performed as shown in Figure 1..$^{19,20}$ Sample distentions were performed, during which the barostat bag was inflated in a stepwise fashion by increasing bag pressure by 4 $\mathrm{mmHg}$ every 15 seconds until the subject reported moderate pain. Then, the bag pressure was set to the individual operating pressure (IOP), ${ }^{26}$ defined as the minimal distending pressure required to overcome mechanical forces and inflate the bag with $30 \mathrm{~mL}$ of air. During this procedure, subjects were placed in the left lateral decubitus position. Perception thresholds in the rectum were assessed using the ascending method of limits. Phasic distensions were 30 seconds in duration and were separated by 30 -second rest intervals, starting at the IOP and increasing by $2 \mathrm{mmHg}$ steps until the subject reported moderate pain (rating of 3 on a $0-5$ pain intensity scale) or pressure reached $48 \mathrm{mmHg}$ without the subject reporting moderate pain (safety limit). The pain threshold was defined as the pressure at which the subject first reported moderate pain (3 on a $0-5$ scale). If the subject did not report moderate pain until 48 $\mathrm{mmHg}$, the pain threshold was assigned a value of $50 \mathrm{mmHg}$. After measuring pain thresholds, there was a 15 -minute rest period.

Phasic contractions were determined from the perfusion ports above and below the bag under the following conditions: (1) during the fasting baseline for 10 minutes at the IOP, (2) during distention for 10 minutes at a pressure of IOP $+20 \mathrm{mmHg}$, (3) during a recovery period after intraluminal distention for 15 minutes at the IOP, and (4) following the meal for 30 minutes at the IOP.

The beginning and ending inflection points for each individual contraction were identified visually and the area under the curve

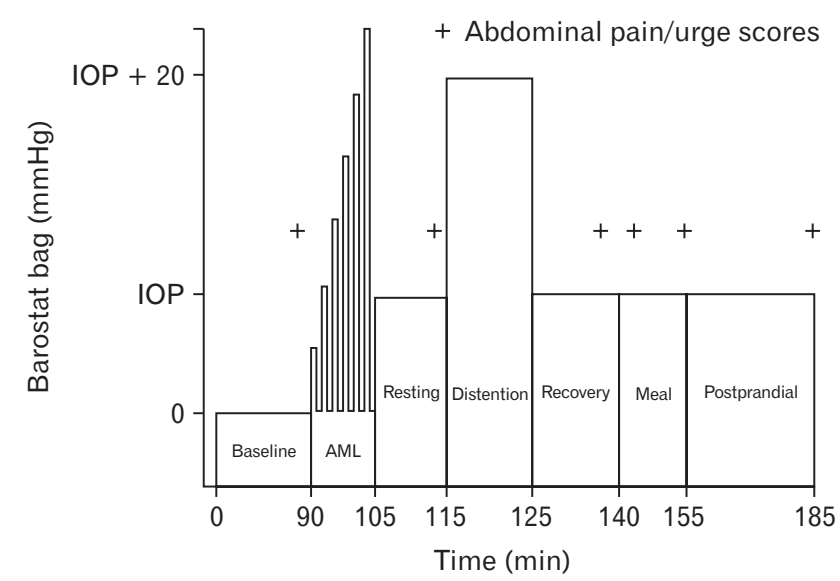

Fiğure 1. Study protocol. IOP, individual operating pressure; AML, ascending method of limits. 
was calculated using a computer software (Polygram, Lower GI Edition, Version 5.06; Medtronic, Inc, Minneapolis, MN, USA). These areas were added together, then divided by recording time in seconds (excluding the time occupied by movement artifact), and multiplied by 100 . The motility index (MI) was the average of phasic contractions at 4 perfusion ports. Colonic smooth muscle tone was defined as the average barostat bag volume during the fasting baseline, in the recovery period after intraluminal distention, and following the meal, using computer software (Sandhill Insight System, Highlands Ranch, CO, USA). ${ }^{26}$ Subjects were asked to consume the meal (standardized content containing 810 kcals including $38 \mathrm{~g}$ of fat) within 10 minutes. After completion of the meal, the subject immediately returned to the left-lateral position and bag pressure was maintained at the IOP for 30 minutes (the postprandial period). Phasic and tonic motility were recorded throughout this period.

\section{Statistical Methods}

Since IBS is considered to be a heterogeneous disorder and HRV in healthy subjects is known to be different by age, the sample size in the present study was determined based on statistical advice in order to perform the multiple regression analysis, and previous studies on HRV in patients with IBS. ${ }^{27,28}$ The variables were analyzed by the non-parametric Mann-Whitney U statistic and the Spearman rank correlation coefficient to account for non-normal distributions of some variables. Categorical data were analyzed with the $\chi^{2}$ test. Linear mixed model was used to compare the HRV values measured across three time periods. The relationships between HRV and physiological and psychological data were evaluated in IBS and control groups, respectively. Multiple linear regression was used to adjust for confounding factors. In addition, the partial correlation analyses that controlled for age within each subgroup (ie, within younger and older IBS patients) were performed when the HRV values were different by age. SPSS version 24.0 (IBM Corp, Armonk, NY, USA) was used for statistical analysis. A $P<$ 0.05 without adjustment for multiple comparisons defined statistical significance.

\section{Results}

\section{Subjects}

One hundred fifty-six IBS patients (131 female; mean age 35 \pm 11 years) and 31 healthy control subjects ( 24 female; $37 \pm 13$ years) were studied.
The IBS cases did not differ from the healthy controls in gender or age. IBS patients had significantly lower scores on the IBSQOL, higher scores on the IBS-SSS, and greater psychological distress on the BSI compared to healthy controls. As previously reported, ${ }^{20}$ IBS patients had significantly lower pain thresholds $(P=$ $0.004)$ and urge thresholds $(P=0.001)$ compared to healthy controls. IBS patients included 32 IBS-C (29 females), 55 IBS-D (42 females), 56 IBS-M (48 females), and 13 IBS-U (12 females). IBS subtype was not significantly related to age or sex.

\section{Effects of Balloon Distention and Eating on Heart Rate Variability}

The $\% \mathrm{HF}$ band power or $\mathrm{LF} / \mathrm{HF}$ ratio was not significantly different during the baseline between IBS and HC groups (Table 1). The Linear mixed model revealed that IBS patients had a significant change in $\mathrm{LF} / \mathrm{HF}$ ratio across the time periods $(P=0.027$, group $\times$ period interaction; Table 1$)$. IBS patients significantly demonstrated less change in $\mathrm{LF} / \mathrm{HF}$ ratio between the baseline and distention periods compared to $\mathrm{HC}(P=0.007$, group $\times$ period interaction). On the other hand, no significant change in $\% \mathrm{HF}$ was observed between IBS and HC across the 3 periods (Table 1). There were no significant differences in any HRV parameters among IBS subtypes.

\section{Effects of Age on Heart Rate Variability}

Among all IBS patients, age significantly negatively correlated with $\% \mathrm{HF}$ and positively correlated with the ratio LF/HF (Fig. 2). These correlations were significant both during baseline (rho $=$ -0.482 for $\% \mathrm{HF}$ band power and rho $=0.492$ for LF/HF; $P<$ 0.001 in both instances), and also during the postprandial period (rho $=-0.348$ for $\% \mathrm{HF}$ band power and rho $=0.359$ for $\mathrm{LF} / \mathrm{HF}$ ratio; $P<0.001$ in both instances).

Table 1. Changes in Heart Rate Variability Parameters

\begin{tabular}{llccc}
\hline \multicolumn{1}{c}{ Variable } & \multicolumn{1}{c}{ Group } & Baseline & Distention & Post-meal \\
\hline$\% \mathrm{HF}^{\mathrm{a}}$ & Controls & $49 \pm 18$ & $38 \pm 18$ & $36 \pm 14$ \\
& IBS & $52 \pm 19$ & $42 \pm 17$ & $39 \pm 16$ \\
$\mathrm{LF} / \mathrm{HF}^{\mathrm{a}, \mathrm{b}}$ & Controls & $1.5 \pm 1.1$ & $2.8 \pm 2.8$ & $2.8 \pm 2.0$ \\
& IBS & $1.7 \pm 2.0$ & $2.0 \pm 1.7^{\mathrm{c}}$ & $2.6 \pm 2.3$ \\
\hline
\end{tabular}

${ }^{\mathrm{a} P}<0.01$, period effect by the linear mixed model across the 3 periods. ${ }^{\mathrm{b}} \mathrm{P}<0.05$, group $\times$ period interaction.

${ }^{\mathrm{c}} P<0.05$, group $\times$ period interaction between the baseline and distention periods.

HF, high frequency; LF, low frequency.

Data are shown as mean $\pm \mathrm{SD}$. 
Healthy subjects as a group showed significant correlations between age and $\mathrm{HRV}$ only during baseline (\% HF band power rho $=-0.407, P=0.023 ; \mathrm{LF} / \mathrm{HF}$ ratio rho $=0.439, P=0.014)$ and not during the postprandial period (\%HF band power rho $=$ $-0.149, P=0.423 ; \mathrm{LF} / \mathrm{HF}$ ratio rho $=0.229, P=0.214)$.

\section{Heart Rate Variability Effects on Colonic Motility and Sensitivity}

During the baseline, neither IBS patients nor healthy controls showed significant correlations between HRV and the MI, the bag volume or the pain/urge threshold. However, during the postprandial period, the postprandial MI significantly correlated with the postprandial $\% \mathrm{HF}$ band power (rho $=-0.231, P=0.006$ ) and $\mathrm{LF} / \mathrm{HF}$ ratio (rho $=0.229, P=0.006$ ) in IBS patients but not in controls (Table 2 and Fig. 3). The multiple regression analyses showed that $\% \mathrm{HF}$ was still a significant negative predictor of postprandial MI after adjusting for age in IBS patients (beta $=-0.242$, $P=0.007)$ but $\mathrm{LF} / \mathrm{HF}$ ratio was not (beta $=0.112, P=0.209$ ).

\section{Heart Rate Variability Effects on Irritable Bowel Syndrome and Psychological Symptoms}

In IBS patients, IBS severity was not correlated with HRV during the baseline, distention, or postprandial period. The general score on the BSI significantly correlated with \% HF (rho $=-0.199$, $P=0.010$ ) and $\mathrm{LF} / \mathrm{HF}$ ratio (rho $=0.206, P=0.013$ ) during the baseline (Table 2). The score for depression on the BSI also significantly correlated with $\% \mathrm{HF}$ (rho $=-0.178, P=0.027$ ) and $\mathrm{LF} / \mathrm{HF}$ ratio (rho $=0.182, P=0.023$ ) during the baseline. Mul-

A

Baseline
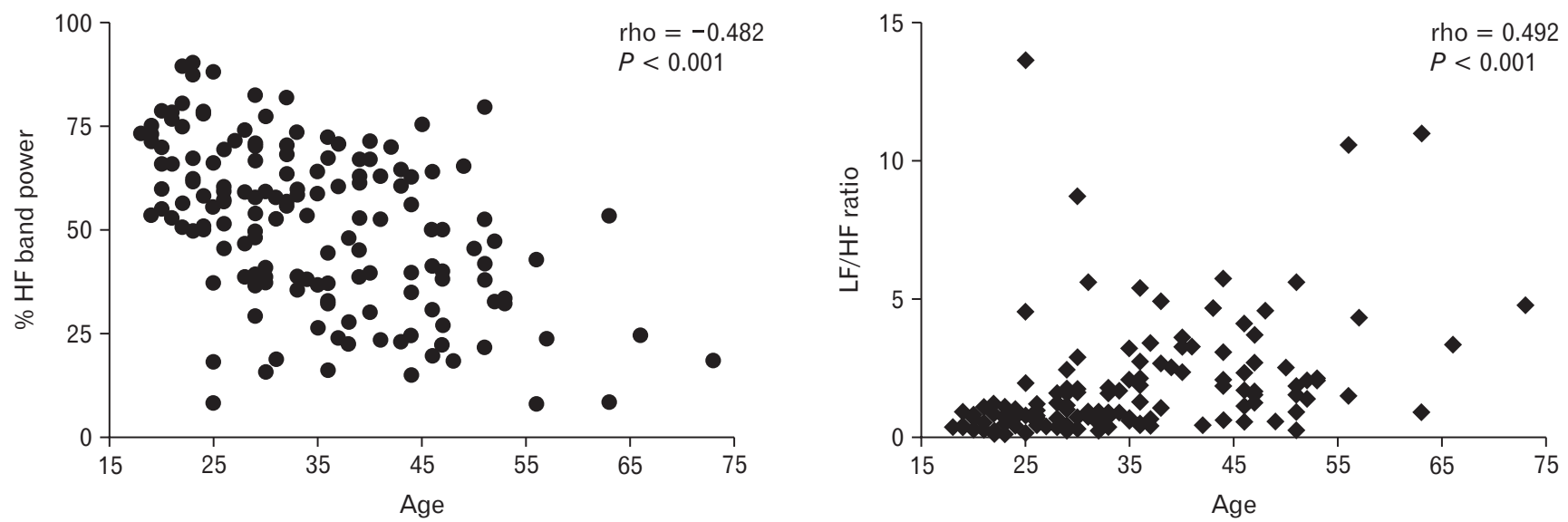

B

Postprandial
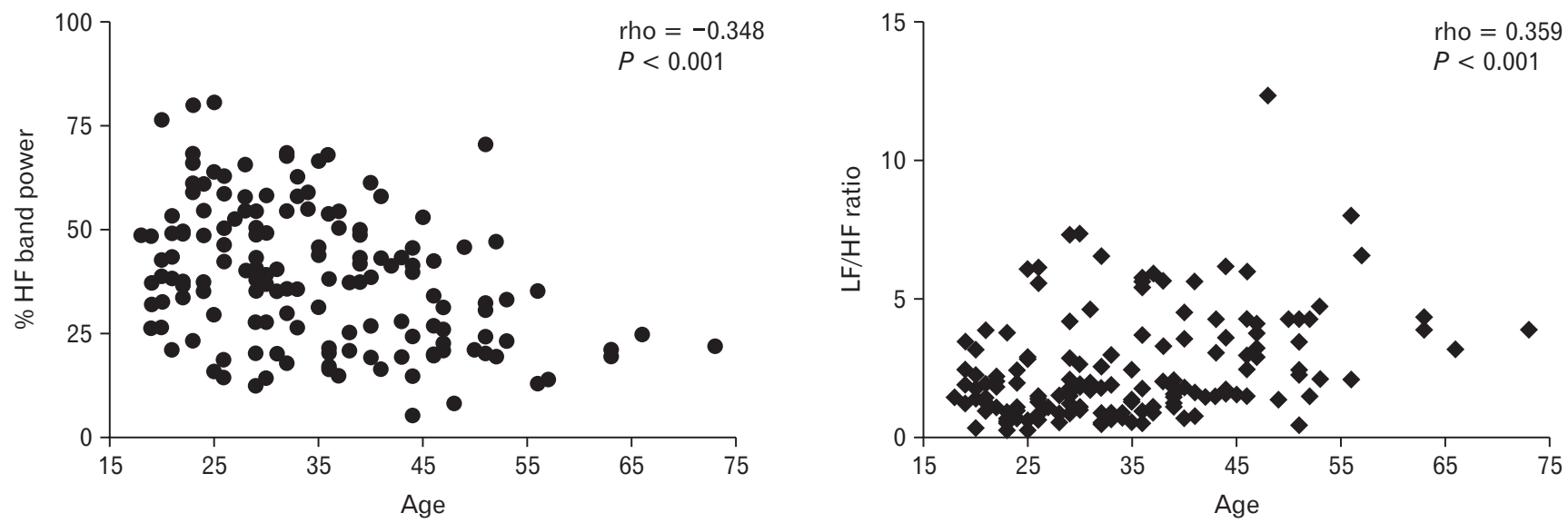

Figure 2. Correlation between age and high frequency (\%HF) band power or low frequency (LF)/HF ratio at baseline and postprandial period in irritable bowel syndrome patients. (A) \% HF band power (left) and $\mathrm{LF} / \mathrm{HF}$ ratio (right) during baseline and (B) \% $\mathrm{HF}$ band power (left) and LF/ HF ratio (right) during postprandial period. 
tiple regression analyses revealed that $\% \mathrm{HF}$ (beta $=-0.221, P=$ 0.018 ) during the baseline was still a significant predictor of general score on the BSI after adjusting for age. On the other hand, significant correlations were no longer found between the other scores on the BSI and HRV parameters after adjusting for age.

In healthy controls, the IBS severity scale or the IBS-QOL

Table 2. Correlations Between Heart Rate Variability Parameters and Physiological and Psychological Data

\begin{tabular}{lccccc}
\hline \multirow{2}{*}{ HRV parameters } & \multicolumn{2}{c}{ Controls $(\mathrm{n}=31)$} & & \multicolumn{2}{c}{ IBS $(\mathrm{n}=156)$} \\
\cline { 2 - 3 } \cline { 5 - 6 } & $\% \mathrm{HF}$ & $\mathrm{LF} / \mathrm{HF}$ & & $\% \mathrm{HF}$ & $\mathrm{LF} / \mathrm{HF}$ \\
\hline Motility index (MI) & & & & \\
$\quad$ Baseline & -0.208 & 0.145 & & -0.098 & 0.102 \\
Distention & 0.266 & -0.250 & & 0.013 & -0.016 \\
$\quad$ Postprandial & 0.003 & 0.067 & & $-0.231^{\mathrm{a}}$ & $0.229^{\mathrm{a}}$ \\
Pain threshold & 0.046 & -0.034 & & 0.003 & 0.004 \\
Urge threshold & -0.329 & 0.231 & & -0.031 & 0.059 \\
IBS-SSS & -0.109 & 0.015 & & 0.018 & -0.011 \\
IBS-QOL & 0.287 & -0.291 & & 0.106 & -0.107 \\
BSI-18 & & & & \\
$\quad$ General & -0.069 & 0.047 & & $-0.199^{\mathrm{b}}$ & $0.206^{\mathrm{b}}$ \\
Somatization & -0.063 & 0.059 & -0.062 & 0.067 \\
Depression & -0.251 & 0.258 & $-0.178^{\mathrm{b}}$ & $0.182^{\mathrm{b}}$ \\
Anxiety & 0.020 & -0.084 & -0.125 & 0.133 \\
\hline
\end{tabular}

${ }^{\mathrm{a}} \mathrm{P}<0.01$.

${ }^{\mathrm{b}} \mathrm{P}<0.05$.

HRV, heart rate variability; HF, high frequency; LF, low frequency; IBSSSS, Irritable Bowel Syndrome Symptom Severity Scale; IBS-QOL, Irritable Bowel Syndrome Quality of Life; BSI, Brief Symptom Inventory.

Data are shown as rho scores by the Spearman rank correlation coefficient. Baseline data for $\% \mathrm{HF}$ or $\mathrm{LF} / \mathrm{HF}$ were used to assess the correlations except motility index during distention and postprandial periods.

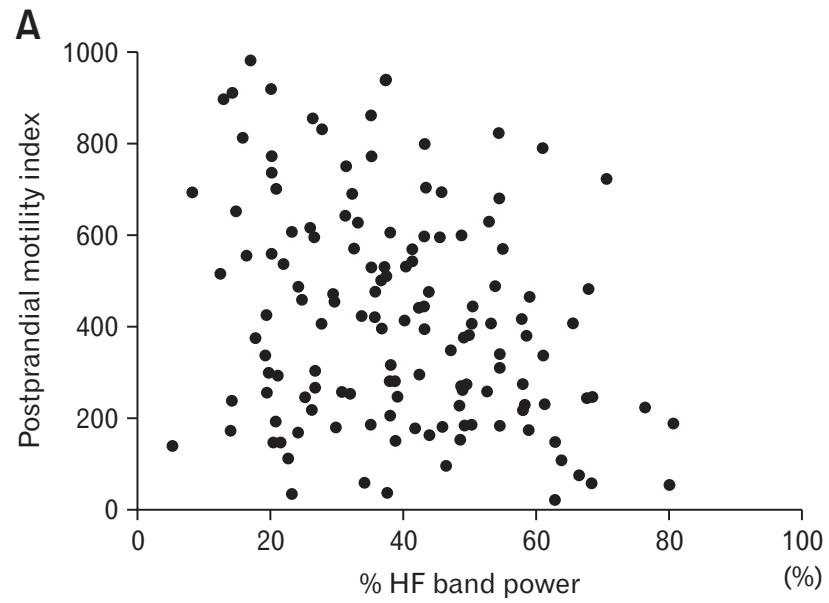

score was not correlated with any $\% \mathrm{HF}$ or $\mathrm{LF} / \mathrm{HF}$ ratio. Each score for on the BSI was not correlated with \% $\mathrm{HF}$ and $\mathrm{LF} / \mathrm{HF}$ ratio during the baseline (Table 2 ).

\section{Post Hoc Analyses of Age Effects on Heart Rate Variability}

To further explore the interactions between age, HRV, and other motility parameters, we divided the IBS group into younger and older cohorts. The cut-off point used to separate younger from older IBS subjects was the average age at which the ratio of LF/ $\mathrm{HF}$ changes from a positive value to a negative one as shown by the crossing of regression lines for $\% \mathrm{LF}$ and $\% \mathrm{HF}$ (Fig. 4). From the findings, the most appropriate cut-off point for age was estimated at 36.5 years. The younger cohort included 95 IBS patients ( $81 \mathrm{fe}-$ males), and the older cohort included 61 IBS patients (50 females). There was no significant difference between younger and older IBS patients in IBS-SSS, IBS-QOL, or any psychological score on the BSI.

In younger IBS patients, the postprandial MI significantly correlated with the postprandial $\% \mathrm{HF}$ band power ( $\mathrm{rho}=-0.359, P$ $=0.001$ ) and LF/HF ratio (rho $=0.330, P=0.002$, Supplementary Table). Multiple linear regression analyses showed that $\% \mathrm{HF}$ (beta $=-0.398, P<0.001$ ) and $\mathrm{L} / \mathrm{H}$ ratio (beta $=0.310, P=$ 0.003 ) during the postprandial period were still significant predictors of postprandial MI after adjusting for age in IBS patients. The general score on the BSI significantly correlated with $\% \mathrm{HF}$ (rho= -0.208, $P=0.043$ ) and $\mathrm{LF} / \mathrm{HF}$ ratio (rho $=0.219, P=0.033$ ) during the baseline. The score for anxiety on the BSI correlated with $\mathrm{LF} / \mathrm{HF}$ ratio (rho $=0.204, P=0.047$ ) during the baseline.

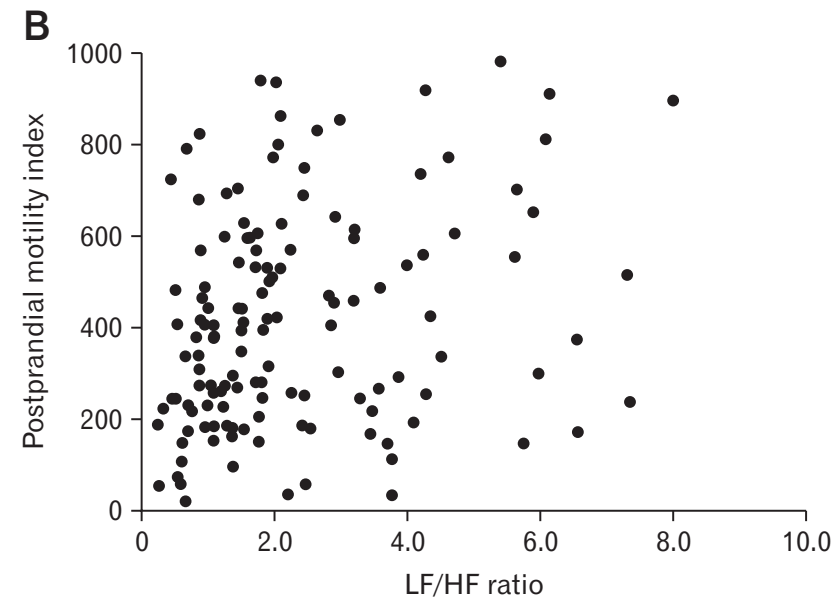

Figure 3. Correlation between heart rate variability (HRV) parameters and colonic motility in irritable bowel syndrome patients. (A) High frequency $(\% \mathrm{HF})$ band power and motility index $($ rho $=-0.231, P=0.006)$ and $(\mathrm{B})$ low frequency $(\mathrm{LF}) / \mathrm{HF}$ ratio and motility index $($ rho $=0.229$, $P=0.006)$ during the postprandial period. 


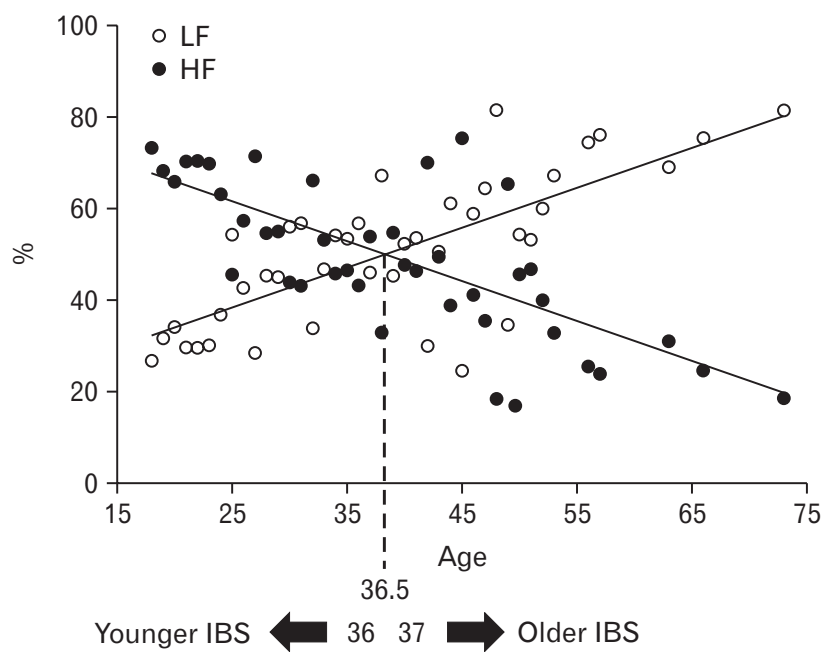

Figure 4. Average score of low frequency (\%LF) and high frequency $(\% \mathrm{HF})$ power scores at baseline in each age. The younger irritable bowel syndrome (IBS): \% HF band power $>\% \mathrm{LF}$ band power, the older IBS: $\% \mathrm{HF}$ band power $<\% \mathrm{LF}$ band power.

After adjusting for age, \%HF (beta $=-0.237, P=0.034$ ) and $\mathrm{L} /$ $\mathrm{H}$ ratio (beta $=0.232, P=0.027$ ) during the baseline were still significant predictors of general score on the BSI. A significant correlation between the anxiety score and $\mathrm{LF} / \mathrm{HF}$ ratio was no longer found after adjusting for age. Pain/urge threshold, the bag volume, the IBS-SSS and BSI psychological scales did not correlate significantly with HRV in the younger cohort.

The older IBS group did not show any significant correlation between HRV and the physiological or psychological parameters (Supplementary Table).

\section{Discussion}

This is the largest study to date of the relationship between autonomic nervous system activity, colonic sensorimotor activity, and symptoms in IBS patients. The key findings are (1) the high frequency band of HRV (\%HF) declines with age both in IBS and healthy subjects, and (2)\%HF negatively correlated with postprandial phasic motility in IBS patients even after adjusting for age. These observations may help to account for the frequent exacerbation of IBS symptoms during the postprandial period. ${ }^{11-13}$

Food intake induces gastric accommodation and stimulates the ANS ${ }^{29,30}$ An increased blood volume after meal ingestion induces vasopressin release through the supraoptic and paraventricular nuclei of the hypothalamus. ${ }^{31}$ The paraventricular nuclei also have neurons releasing corticotrophin-releasing hormone and noradrena- line. ${ }^{32}$ These peptides play an important role not only in homeostasis but also in stress responses. Simrén et al showed that IBS patients have colonic hypersensitivity during duodenal infusion of lipids. ${ }^{33}$ We confirmed sympathetic arousal during the postprandial period, which is reflected in a higher LF/HF ratio in young IBS patients. The findings in our study suggest an intrinsic role of the autonomic nervous system from enteric neurons to spinal afferent nerves after meal ingestion and its dysfunction in IBS patients. We also found that withdrawal of parasympathetic activity and increased sympathetic activity correlates with colonic motility during the postprandial period. In IBS patients, meal ingestion often causes increased colonic motility which accelerates colonic transit. ${ }^{19}$

This study shows that HRV is related to age. The HF band is greater than the LF band in the younger IBS group whereas the opposite is true in the older IBS group (Fig. 4). Population-based studies in healthy subjects also supported age-related changes in $\mathrm{HF}$ but not in $\mathrm{LF} / \mathrm{HF}$ ratio. ${ }^{14-16} \mathrm{HF}$ power increases 5 -fold during the first two decades of life, then it begins to decline. Between the ages 30 and 40 years $\% \mathrm{HF}$ shows the most marked decline. ${ }^{15}$ In this study, those in the younger group who showed higher MI after meal ingestion have lower \% $\mathrm{HF}$ and higher $\mathrm{LF} / \mathrm{HF}$ ratio. Healthy subjects over 40 years old showed a smaller decrease in $\mathrm{HF}$ as well as a decreasing Valsalva ratio. ${ }^{34}$ Saito et al $^{3}$ reported the prevalence of IBS as $13.5 \%$ in under 45 seconds and $9.4 \%$ in over 45 seconds. We speculate that the decline in IBS symptoms as people age may be influenced by the reduced activity of the ANS.

Our study did not show significant HRV differences between IBS patients and healthy controls during any study period although IBS patients showed a significant blunting sympathetic response to the colonic distention. Some previous reports have also failed to show a significant $\mathrm{HF}$ and $\mathrm{LF} / \mathrm{HF}$ ratio between IBS patients and controls in response to meal or water ingestion. ${ }^{35,36}$ Our study suggests that $\mathrm{HRV}$ in response to meal ingestion is different depending on age in both IBS patients and healthy subjects, thus, the distribution of subjects' age might cause significant HRV differences. Another possibility is that cardiac vagal efferent nerve activity was not altered in IBS patients during the resting state. Interestingly, IBS patients significantly demonstrated less change in LF/HF ratio (ie, cardiosympathetic balance) from the baseline to colonic distention periods compared to controls in the present study (Table 1). It has been reported that IBS patients had significantly less cardiosympathetic and cardiovagal responsiveness after controlling age effect during flexible sigmoidoscopy which may cause visceral nociception. ${ }^{28}$ The altered responses in the emotional-arousal network during visceral stimulation were shown in IBS patients by means of 
brain imaging techniques. ${ }^{37-39}$

These brain regions are closely related to stress responses which often induce cardiovascular activation. However, meal stimulation may not directly affect the brain regions which mediate pathophysiological conditions of IBS. Thus, the differences of HRV responses between IBS patients and healthy controls in some studies might be caused by the study design. However, younger IBS patients showed significant correlation between postprandial HRV, pain threshold and IBS-QOL. These data therefore suggest that ANS in younger IBS patients may represent the altered neural pathways between gastro-colonic reflexes and the sensory component.

This study has some limitations. First, blood glucose and lipid levels were not monitored when assessing the response to eating. These changes in metabolism may affect colonic motility, vascular regulation, or emotional state. Total meal calories may also affect colonic motility, but variations in calories consumed are unlikely to contribute to error variance in this study because subjects consumed a standard high-fat meal. Second, the number of male subjects was too small to test for sex differences. Tillisch et $\mathrm{al}^{40}$ reported sex differences in ANS in IBS patients. However, our findings suggest that age may have a greater impact on HRV than does sex. ${ }^{41-43}$ Third, as the post-hoc analyses, we set 37 years to divide the young and old age groups based on the ratio of LF/HF changes by age, which might be biased. However, misleading conclusions seem to be unlikely since the postprandial motor activity significantly correlated with the postprandial \% HF even in all IBS patients (Fig. 3). Imam et $\mathrm{al}^{44}$ previously proposed that $\mathrm{QT}$ and $\mathrm{RR}$ variability may be different among young (20-35 years), middle-aged (4055 years), and elderly (above 60 years) subjects. Further validation studies should be warranted to confirm the cut-off point by age on autonomic nervous function in patients with IBS.

In conclusion, our results suggest that, decreased vagal activity is associated with increase in age and greater postprandial colonic motility in patients with IBS, which may contribute to postprandial symptoms.

\section{Supplementary Material}

Note: To access the supplementary table mentioned in this article, visit the online version of Journal of Neurogastroenterology and Motility at http://www.jnmjournal.org/, and at https://doi. org/10.5056/jnm16216.

Financial support: This study was supported by the National Institute of Diabetes and Digestive and Kidney Diseases (Grant

\section{No. R01 DK031369).}

Conflicts of interest: William E Whitehead receives grant support from Ironwood, Takeda, and Salix pharmaceutical companies for studies unrelated to the current manuscript. Other authors have nothing to disclose with relation to the current study.

Author contributions: Yukari Tanaka: data analysis and preparation of the manuscript; Motoyori Kanazawa: participated in study design, data analysis, and preparation of the manuscript; Olafur S Palsson: participated in study design and data analysis; Miranda A Van Tilburg: participated in data collection and data analysis; Lisa M Gangarosa: participated in data collection; Shin Fukudo: participated in data analysis and preparation of the manuscript: Douglas A Drossman: participated in study design and data collection; and William E Whitehead: the guarantor of this paper, participated in study design, data collection, data analysis, and manuscript preparation.

\section{References}

1. Longstreth GF, Thompson WG, Chey WD, Houghton LA, Mearin F, Spiller RC. Functional bowel disorders. Gastroenterology 2006;130:1480-1491.

2. Gwee KA, Bak YT, Ghoshal UC, et al. Asian consensus on irritable bowel syndrome. J Gastroenterol Hepatol 2010;25:1189-1205.

3. Saito YA, Schoenfeld P, Locke GR 3rd. The epidemiology of irritable bowel syndrome in North America: a systematic review. Am J Gastroenterol 2002;97:1910-1915.

4. Hyams JS, Burke G, Davis PM, Rzepski B, Andrulonis PA. Abdominal pain and irritable bowel syndrome in adolescents: a community-based study. J Pediatr 1996;129:220-226.

5. Whitehead WE, Crowell MD, Robinson JC, Heller BR, Schuster MM. Effects of stressful life events on bowel symptoms: subjects with irritable bowel syndrome compared with subjects without bowel dysfunction. Gut 1992;33:825-830.

6. Tanaka Y, Kanazawa M, Fukudo S, Drossman DA. Biopsychosocial model of irritable bowel syndrome. J Neurogastroenterol Motil 2011;17:131-139.

7. Malik M, Bigger JT, Camm AJ, et al. Heart rate variability standards of measurement, physiological interpretation, and clinical use. Eur Heart J 1996;17:354-381.

8. Mayer EA, Naliboff BD, Chang L, Coutinho SV. V. Stress and irritable bowel syndrome. Am J Physiol Gastrointest Liver Physiol 2001;280:G519-G524.

9. Elsenbruch S, Orr WC. Diarrhea-and constipation-predominant IBS patients differ in postprandial autonomic and cortisol responses. Am J Gastroenterol 2001;96:460-466.

10. Ng C, Malcolm A, Hansen R, Kellow J. Feeding and colonic distension provoke altered autonomic responses in irritable bowel syndrome. Scand J Gastroenterol 2007;42:441-446. 
11. Marciani L, Cox EF, Hoad CL, et al. Postprandial changes in small bowel water content in healthy subjects and patients with irritable bowel syndrome. Gastroenterology 2010;138:469-477.e1.

12. Di Stefano M, Miceli E, Missanelli A, Mazzocchi S, Corazza GR. Meal induced rectosigmoid tone modification: a low caloric meal accurately separates functional and organic gastrointestinal disease patients. Gut 2006;55:1409-1414.

13. Ludidi S, Conchillo JM, Keszthelyi D, et al. Does meal ingestion enhance sensitivity of visceroperception assessment in irritable bowel syndrome? Neurogastroenterol Motil 2012;24:47-53, e3.

14. Antelmi I, de Paula RS, Shinzato AR, Peres CA, Mansur AJ, Srupi CJ. Influence of age, gender, body mass index, and functional capacity on heart rate variability in a cohort of subjects without heart disease. Am J Cardiol 2004;93:381-385.

15. Korkushko OV, Shatilo VB, Plachinda Yul, Shatilo TV. Autonomic control of cardiac chronotropic function in man as a function of age: assessment by power spectral analysis of heart rate variability. J Auton Nerv Syst 1991;32:191-198.

16. O'Brien IA, O'Hare P, Corrall RJ. Heart rate variability in healthy subjects: effect of age and the derivation of normal ranges for tests of autonomic function. Br Heart J 1986;5 5:348-354.

17. Kelly J, O'malley K. Adrenoceptor function and ageing. Clin Sci (Lond) 1984;66:509-515.

18. Hungin AP, Whorwell PJ, Tack J, Mearin F. The prevalence, patterns and impact of irritable bowel syndrome: an international survey of 40,000 subjects. Aliment Pharmacol Ther 2003;17:643-650.

19. Dorn SD, Palsson OS, Thiwan SI, et al. Increased colonic pain sensitivity in irritable bowel syndrome is the result of an increased tendency to report pain rather than increased neurosensory sensitivity. Gut 2007;56:12021209.

20. Kanazawa M, Palsson OS, Thiwan SI, et al. Contributions of pain sensitivity and colonic motility to IBS symptom severity and predominant bowel habits. Am J Gastroenterol 2008;103:2550-2561.

21. Adeyemi EO, Desai KD, Towsey M, Ghista D. Characterization of autonomic dysfunction in patients with irritable bowel syndrome by means of heart rate variability studies. Am J Gastroenterol 1999;94:816-823.

22. Patrick DL, Drossman DA, Frederick IO, DiCesare J, Puder KL. Quality of life in persons with irritable bowel syndrome: development and validation of a new measure. Dig Dis Sci 1998;43:400-411.

23. Francis CY, Morris J, Whorwell PJ. The irritable bowel severity scoring system: a simple method of monitoring irritable bowel syndrome and its progress. Aliment Pharmacol Ther 1997;11:395-402.

24. Derogatis LR. BSI 18 Brief symptom inventory 18: Administration, scoring, and procedures manual, Minneapolis: NCS Pearson, Incorporated 2000.

25. Buchman TG, Stein PK, Goldstein B. Heart rate variability in critical illness and critical care. Curr Opin Crit Care 2002;8:311-315.

26. Whitehead WE, Delvaux M. Standardization of barostat procedures for testing smooth muscle tone and sensory thresholds in the gastrointestinal tract. The Working Team of Glaxo-Wellcome Research, UK. Dig Dis Sci 1997;42:223-241.

27. Jarrett ME, Burr RL, Cain KC, Rothermel JD, Landis CA, Heit- kemper MM. Autonomic nervous system function during sleep among women with irritable bowel syndrome. Dig Dis Sci 2008;53:694-703.

28. Cheng P, Shih W, Alberto M, et al. Autonomic response to a visceral stressor is dysregulated in irritable bowel syndrome and correlates with duration of disease. Neurogastroenterol Motil 2013;25:e650-e659.

29. Berthoud HR. The vagus nerve, food intake and obesity. Regul Pept 2008;149:15-25.

30. Takahashi T, Owyang C. Characterization of vagal pathways mediating gastric accommodation reflex in rats. J Physiol 1997;504(Pt 2):479-488.

31. Bisset GW, Chowdrey HS. Control of release of vasopressin by neuroendocrine reflexes. Q J Exp Physiol 1988;73:811-872.

32. Chrousos GP. Stressors, stress, and neuroendocrine integration of the adaptive response. The 1997 Hans Selye Memorial Lecture. Ann N Y Acad Sci 1998;851:311-335.

33. Simrén M, Abrahamsson H, Björnsson ES. Lipid-induced colonic hypersensitivity in the irritable bowel syndrome: the role of bowel habit, sex, and psychologic factors. Clin Gastroenterol Hepatol 2007;5:201-208.

34. Ramaekers D, Ector H, Aubert AE, Rubens A, Van de Werf F. Heart rate variability and heart rate in healthy volunteers. Is the female autonomic nervous system cardioprotective? Eur Heart J 1998;19:13341341.

35. Elsenbruch S, Lovallo WR, Orr WC. Psychological and physiological responses to postprandial mental stress in women with the irritable bowel syndrome. Psychosom Med 2001;63:805-813.

36. Jarrett ME, Han CJ, Cain KC, et al. Relationships of abdominal pain, reports to visceral and temperature pain sensitivity, conditioned pain modulation, and heart rate variability in irritable bowel syndrome. Neurogastroenterol Motil 2016;28:1094-1103.

37. Mertz H, Morgan V, Tanner G, et al. Regional cerebral activation in irritable bowel syndrome and control subjects with painful and nonpainful rectal distention. Gastroenterology 2000;118:842-848.

38. Mayer EA, Berman S, Suyenobu B, et al. Differences in brain responses to visceral pain between patients with irritable bowel syndrome and ulcerative colitis. Pain 2005;115:398-409.

39. Rapps N, van Oudenhove L, Enck P, Aziz Q. Brain imaging of visceral functions in healthy volunteers and ibs patients. J Psychosom Res 2008;64:599-604.

40. Tillisch K, Mayer EA, Labus JS, Stains J, Chang L, Saliboff BD. Sex specific alterations in autonomic function among patients with irritable bowel syndrome. Gut 2005;54:1396-1401.

41. Jensen-Urstad K, Storck N, Bouvier F, Ericson M, Lindblad LE, Jensen-Urstad H. Heart rate variability in healthy subjects is related to age and gender. Acta Physiol Scand 1997;160:235-241.

42. Zhang J. Effect of age and sex on heart rate variability in healthy subjects. J Manipulative Physiol Ther 2007;30:374-379.

43. Umetani K, Singer DH, McCraty R, Atkinson M. Twenty-four hour time domain heart rate variability and heart rate: relations to age and gender over nine decades. J Am Coll Cardiol 1998;31:593-601.

44. Imam MH, Karmakar CK, Palanaiswami M, Khandoker AH. A novel technique to investigate the effect of ageing on ventricular repolarization characteristics in healthy and LQTS subjects. Conf Proc IEEE Eng Med Biol Soc 2015:2015;2796-2799. 